\title{
REDISTRIBUTIVE TAXATION AS SOCIAL INSURANCE
}

\author{
Hal R. VARIAN* \\ University of Michigan, Ann Arbor, MI 48109, USA
}

Received October 1979, revised version received March 1980

\begin{abstract}
The modern literature on nonlinear optimal taxation treats differences in income as being due to unobserved differences in ability. A striking result of this assumption is that high income agents should face a zero marginal tax rate. In this paper I assume that differences in observed income are due to exogenous differences in luck. Hence the optimal redistributive tax involves trading off the benefits due to 'social insurance' with the costs due to reduced incentives. I derive the optimal forms for linear and nonlinear taxes, and compute some algebraic and numeric examples. Typically high income individuals will face quite high marginal tax rates.
\end{abstract}

\section{Introduction}

Recent work on the sources of income inequality has found that only a small fraction of the variation in income can be explained by observed socioeconomic characteristics. For example, after an extensive examination of the available evidence, Jencks and his co-workers conclude: 'Neither family background, cognitive skill, educational attainment, nor occupational status explains much of the variation in men's income. Indeed when we compare men who are identical in all these respects, we find only 12 to 15 percent less inequality than among random individuals' [Jencks (1972, p. 266)].

Jencks goes on to list three possible causes for such unexplained variations in income.

(1) Differences in tastes: '... some men value money more than others, and these men make unusual sacrifices to get it'.

(2) Differences in unobserved endowments of native ability: ‘... the ability to hit a ball thrown at high speed, the ability to type a letter quickly and accurately, the ability to persuade a customer that he wants a larger car than he thought he wanted... and so forth'.

(3) Differences in luck: '. . chance acquaintances who steer you to one line of work rather than another, the range of jobs that happens to be available in a particular community when you are job hunting, the amount of

*I have benefited from comments by Theodore Bergstrom, Lamberto Cesari, Bengt Holmström, Glenn Loury, James Mirrlees, Carl Simon and the anonymous referees of this journal. I am of course responsible for any remaining errors. This material is based upon work supported by the National Science Foundation under Grant SOC78-05757 and the Guggenheim Memorial Foundation. 
overtime work in your particular plant, whether bad weather destroys your strawberry crop ... and a hundred other unpredictable accidents'.

Jencks and his co-workers argue that this last factor is probably the most important single influence on the distribution of income. It is difficult to support this view by appealing only to cross-sectional data since one cannot adequately control for variations in unobserved tastes and endowments. However, other econometric studies of income determination using panel data have tended to confirm Jencks's view. Coe (1977), for example, describes some interesting figures which were extracted from the University of Michigan income dynamics study of 5000 American families:

Of the individuals who were poor in the one year period 1975 , only 12 percent were in poverty in every one of the nine years between 1967 and 1975. On the other hand, while only 8.9 percent of the population was poor in 1975, fully one-quarter of the sample individuals were in poverty in at least one of the nine years between 1967 and 1975.

Similar conclusions were reached by Lillard and Willis (1978) who used the University of Michigan income dynamics data for the years 1967-73:

While the poor are different in the sense just described, it would be misleading to conclude that poverty is a permanent status. We find that of those individuals in poverty in a given year, about 55 percent of the whites and 35 percent of the blacks will be out of poverty in the following year. Another indication of mobility is that only 15 percent of whites and 35 percent of blacks who fall into poverty sometime during the three year period from 1967-70 are expected to be in poverty in all three years ( $\mathrm{p}$. 1007).

These data suggest that the movements of individual incomes over time contain a large random component, i.e. a component that is not explained by differences in tastes and endowments.

Such a view tends to modify attitudes concerning policy towards income distribution, and in particular, policies concerned with redistributive taxation. Most discussions of redistributive taxation have taken place in a context where uncertainty was either ignored, or where markets for transferring risk were sufficiently well developed so that no risk was borne unintentionally. The above empirical evidence shows that randomness in income is a major problem which cannot simply be ignored, and well-known arguments concerning moral hazard, adverse selection, transactions costs, and returns to scale show why complete markets for shifting risks may be unavailable.

Let us consider then how adding a random component to income may affect the analysis of redistributive programs. Suppose that we consider a simple world of identical risk-averse individuals each with income $Y_{i}=\vec{Y}+\varepsilon_{i}$. Here $\bar{Y}$ is the expected income of each individual and $\varepsilon_{i}$ is a random error 
term assumed to be uncorrelated between individuals, and to have expected value of zero. Since $\varepsilon_{i}$ is uncorrelated between individuals, there is a clear economic case for the establishment of an insurance market which could eliminate individual risk. However, let us suppose that effects such as those described above are present so that an insurance market cannot be viable. Then there is still a simple government policy to improve welfare: impose a 100 percent tax on observed income and redistribute a uniform grant $\bar{Y}$. Such a program of redistributive taxation would effectively replace the nonexistent insurance market and provide an unambiguous increase in welfare. The motive for redistribution here is not a desire for equity per se, but rather a desire for social insurance.

The above points are couched in somewhat abstract language, but it is clear that the basic idea is commonplacc. Indecd, the fact that redistributive taxation helps to insure against individual risk is a common justification for redistributive programs. Consider, for example, the following excerpt from the Beveridge Report, a study which provided the guidelines for British welfare policy in the post-war period [Beveridge (1942)]:

Abolition of want cannot be brought about merely by increasing production, without seeing to correct distribution of the product... Better distribution of purchasing power is required among wage earners themselves, as between times of earning and not earning, and between times of heavy family responsibilities and light or no family responsibilities. Both social insurance and children's allowances are primary methods of redistributing wealth. (Emphasis added.)

Similar sentimates are expressed by Stanford G. Ross, the current Commissioner of U.S. Social Security: "Everybody is vulnerable to a sudden reduction in income, regardless of his or her station in life. Society needs some mechanism to guard against the risk when it is most likely to occur..." [Ross (1979)].

Jencks and his co-workers have suggested that the most effective way to provide a more equal distribution of income would be to engage in a widespread system of redistributive taxation. The above discussion indicates that even apart from the possibly desirable effect of reducing income inequality, there is a definitely desirable effect of improving the allocation of risk bearing. Indeed, I suspect that widespread political support of many redistributive programs rests more with the social insurance aspect of the program than with altruistic consideration involving social welfare.

Of course, there is a cost to any program of redistributive taxation, namely the incentive loss that is involved in any tax or transactions. The choice of an optimal redistributive tax therefore involves trading off three kinds of effects: (1) the equity effect of changing the distribution of income; (2) the 
efficiency effect from reducing incentives; and (3) the insurance effect from reducing the variance of individual income streams.

The first two aspects have been examined extensively in recent years. Some relevant articles are those by Atkinson and Stiglitz (1976), Diamond (1975), Diamond and Mirrlees (1971), and Mirrlees (1971). Sandmo (1976) provides a brief survey with bibliographic references.

The insurance effect of income redistribution has received considerably less attention in the theoretical literature.

A notable exception has been the work of Mirrlees and his collaborators. Mirrlees (1974) posed the problem of the insurance-incentive tradeoff in a fairly general model and derived a formula characterizing the optimal (second-best) policy. Subsequently, Diamond and Mirrlees (1978) analyzed a model of a Social Security type program, emphasizing the insuranceincentive aspects involved in the retirement decision. Diamond, Helms and Mirrlees (1978) present some numerical examples of social insurance benefits in some simple cases involving linear taxation.

In this paper I formulate and analyze a simple model of social insurance. Section 2 describes the basic model. In section 3 I derive a formula characterizing the optimal linear tax for this model. Section 4 is devoted to an algebraic and numerical example of the linear tax. Section 5 describes the optimal nonlinear tax, and section 7 provides a brief summary. An appendix describes an existence theorem for a class of optimization problems relevant to social insurance problems.

\section{A problem in social insurance}

Let us consider a simple model involving social insurance. We have a large number of identical consumers who wish to transfer some amount of wealth $(w)$ between two time periods. We let $x$ be the amount saved in the first time period so that consumption at that time is $w-x$. Consumption in the second period will be $x+\varepsilon$, where $\varepsilon$ is a random variable with mean zero. The random variable $\varepsilon$ is supposed to represent the cumulative effect of exogenous 'luck' on the individual consumer. We suppose luck to be independently and identically distributed among the individual agents. Since luck is assumed to be independently distributed from consumer to consumer, there is a clear market incentive to provide insurance.

In this framework, the individual optimization problem is:

$$
\max _{x} u(w-x)+\mathrm{E} u(x+\varepsilon)
$$

where $u(y)$ is a von Neuman-Morgenstern utility function and $\mathrm{E}$ is the expectation operator. We assume that $u(y)$ exhibits the standard properties of monotonicity in income and risk aversion. At times we will assume that the utility function exhibits declining absolute risk aversion. These three behavioral assumptions imply $u^{\prime}(y)>0, u^{\prime \prime}(y)<0$, and $u^{\prime \prime \prime}(y)>0$. 
If no insurance were available the individual choice of $x$ would satisfy

$$
\mathrm{u}^{\prime}(\mathrm{w}-\mathrm{x})=\mathrm{E} u^{\prime}(x+\varepsilon)
$$

If we assume $u^{\prime \prime \prime}(y)>0$, Jensen's inequality implies $E u^{\prime}(x+\varepsilon)>u^{\prime}(x)$, and hence $x$ will be greater than $w / 2$. Since no insurance is available, consumers will be forced to self insure by oversaving. If consumers could purchase insurance against the cumulative random events described by $\varepsilon$, they would clearly be able to eliminate all individual risk. If all individual risk is eliminated the obvious individual, and hence social, optimum is to set $x$ $=w / 2$.

On the other hand, there may be various difficulties with observing realizations of luck directly. We assume that an external observer can observe only measured income $y=x+\varepsilon$. Some part of measured income is due to a decision by the individual agent $(x)$, and some part is due to exogenous luck $(\varepsilon)$. For practical reasons, any compensation must be based on observed values of $y=x+\varepsilon$ rather than on $\varepsilon$ alone.

We let $c(x+\varepsilon)$ be the amount of consumption allowed to a consumer with observed income $x+\varepsilon$. The objective of a social insurance program is to find a consumption function that maximizes expected utility subject to two constraints. The first constraint is the government budget constraint: the receipts of the tax must, on the average, be equal to the amount payed out. That is to say, the redistributive program must break even. The second constraint is that individual consumers choose $x$ so as to maximize private utility:

$$
\max _{x} u(w-x)+\operatorname{E} u(c(x+\varepsilon))
$$

This immcdiately implics that the social optimum will be unattainable by a redistributive tax based on observed income: if consumers were guaranteed a second period income of $w / 2$, and a marginal tax rate of 1 on any income in excess of $w / 2$, they would choose to save nothing. This is simply the disincentive effect of social insurance - a complete welfare state may result in a significant incentive loss.

An optimal choice of $x>0$ satisfies the first- and second-order conditions:

$$
\begin{aligned}
& u^{\prime}(w-x)=\mathrm{E} u^{\prime}(c(x+\varepsilon)) c^{\prime}(x+\varepsilon) \\
& u^{\prime \prime}(w-x)+\mathrm{E} u^{\prime \prime}(c(x+\varepsilon)) c^{\prime}(x+\varepsilon)+\mathrm{E} u^{\prime}(c(x+\varepsilon)) c^{\prime \prime}(x+\varepsilon) \geqq 0
\end{aligned}
$$

The first-order condition is simply the statement that the consumer will equate his expected marginal utility of income in each period. We note the first two terms of the second-order condition are automatically negative by 
the assumption of risk aversion. The third term is unfortunately of ambiguous sign since it depends on the sign of $c^{\prime \prime}(x+\varepsilon)$. Our approach here will be to assume that the first-order condition completely describes the optimal choice of the consumer, solve for the optimal $c(y)$, and then verify that such a $c(y)$ satisfies the second-order conditions. As we shall see, for a class of important examples $c^{\prime \prime}(y)$ will turn out to have the correct sign. ${ }^{1}$

Formulating the above discussion algebraically, we have the social insurance problem:

$$
\begin{aligned}
& \max _{c(\cdot), x} u(w-x)+\mathrm{E} u(c(x+\varepsilon)) \\
& \mathrm{E} c(x+\varepsilon)=x, \\
& u^{\prime}(w-x)-\mathrm{E}^{\prime}(c(x+\varepsilon)) c^{\prime}(x+\varepsilon)=0 .
\end{aligned}
$$

We investigate this problem in two stages. First we examine the determination of the optimal linear tax, i.e. where $c(y)=c y+D$. Then we investigate the general problem of the optimal nonlinear tax. ${ }^{2}$

\section{The optimal linear tax}

It is possible to derive the optimal linear tax by differentiating (5) (7) and manipulating the resulting conditions. However, a somewhat clearer derivation results if we proceed by a more indirect route.

The first step is to eliminate the governmental budget constraint. We write after tax income as $c y+D$. According to (6), and that fact that $\mathrm{E} \varepsilon=0$ :

$$
\mathrm{E}\lfloor c y+D\rfloor=\mathrm{E}[c(x+\varepsilon)+D]=c x+D=x .
$$

Thus,

$$
D=(1-c) x
$$

The next step is to use the individual optimization constraint to define $x$ as a function of $c$, which we write as $x(c) .{ }^{3}$ We now have a straightforward

${ }^{1}$ The conditions (3) and (4) are necessary and sufficient conditions for a locally optimal choice of $x$. It may happen that there are several solutions of (3) with only some of them being global optima. Mirrlees (1975) has shown that this may cause difficulties for the above technique.

The problem arises because the set of global optima - the set of solutions to problem (2) can be a rather unpleasantly structured subset of the manifold described by (3). Mirrlees (1975) suggests some techniques to handle these difficulties in the finite dimensional case, but there seem to be no useful techniques available in the infinite dimensional case. Hence, we adopt the above approach and simply assume that the first-order condition uniquely determines the optimal choice.

${ }^{2}$ Note that when we restrict the optimal tax to be linear the second-order conditions (4) are automatically satisfied and the problem mentioned in footnote 1 does not arise.

${ }^{3} \mathrm{~A}$ standard application of the implicit function theorem shows that $x(c)$ is a differentiable function of $c$. This derivative is given in eq. (14). 
unconstrained maximization problem which determines the optimal tax:

$$
\max _{c} u[w-x(c)]+\mathrm{E} u[c(x(c)+\varepsilon)+(1-c) x(c)]
$$

The derivative of the objective function is:

$$
\begin{aligned}
v^{\prime}(c)= & -u^{\prime}(w-x) x^{\prime}(c)+E u^{\prime}(x+c \varepsilon) \\
& \times\left[c x^{\prime}(c)+x+\varepsilon+(1-c) x^{\prime}(c)-x\right] .
\end{aligned}
$$

Applying the individual first-order conditions (3), we can eliminate the first term and part of the second, leaving

$$
v^{\prime}(c)=\mathrm{E} u^{\prime}(x+c \varepsilon)(1-c) x^{\prime}(c)+\mathrm{E} u^{\prime}(x+c \varepsilon) \varepsilon .
$$

We note first that $\mathrm{E} u^{\prime}(x+c \varepsilon) \varepsilon<0$. Since $\mathrm{E} \varepsilon=0$, this term is simply the covariance of marginal utility and income; since these magnitudes move in opposite directions this covariance must be negative.

If we evaluate expression (11) at $c=1$ - the no-tax situation - the first term drops out and we are left with a strictly negative value for $v^{\prime}(c)$. Hence a decrease in $c$ - an increase in the tax rate - must necessarily increase utility. This is simply the point that the first small imposition of the tax will result in no incentive loss, but will provide a small amount of insurance. Therefore some amount of social insurance is desirable, even in the face of the incentive problem.

Setting $v^{\prime}(c)$ equal to zero we have a formula for the optimal tax:

$$
\mathrm{E} u^{\prime}(x+c \varepsilon)(1-c) x^{\prime}(c)+\mathrm{E} u^{\prime}(x+c \varepsilon) \varepsilon=0 .
$$

In order to provide some intuition for this expression we imagine an experiment of increasing $c$ slightly to $c+\Delta c$. On the one hand an increase in $c$ will result in more savings, since consumers get to keep more of their returns - savings go up by $x^{\prime}(c) \Delta c$. (Eq. (11) shows that $x^{\prime}(c)$ is positive at the optimal $c$.) Because of this increase in savings, consumers will receive an increase in the demogrant $D$ of $(1-c) x^{\prime}(c) \Delta c$. This in turn is multiplied by the marginal utility of second-period income to determine the utility effect of this change. On the other hand, the increase in $c$ exposes the consumer to more risk. Instead of facing a random variable $c \varepsilon$, the consumer faces $(c+\Delta c) \varepsilon$. The expected marginal utility due to this effect is $\mathrm{E} u(x+c \varepsilon) \varepsilon \Delta c$. If the level of social insurance is optimal, the sum of these two effects must balance out to zero. 
Eq. (11) can be combined with the individual first-order conditions to give:

$$
c=\frac{u^{\prime}(w-x) x^{\prime}(c)}{u^{\prime}(w-x) x^{\prime}(c)-\mathrm{E} u^{\prime}(x+c \varepsilon) \varepsilon} .
$$

This equation shows that the optimal marginal tax rate must be between 0 and 1.

We can derive an expression for the term $x^{\prime}(c)$ by differentiating the firstorder conditions which define $x$. We find

$$
u^{\prime \prime}(w-x) x^{\prime}(c)+c \mathrm{E} u^{\prime \prime}(x+c \varepsilon)\left[x^{\prime}(c)+\varepsilon\right]+\mathrm{E} u^{\prime}(x+c \varepsilon)=0
$$

which can be rearranged to give

$$
x^{\prime}(c)=\frac{-\mathrm{E} u^{\prime \prime}(x+c \varepsilon) c \varepsilon-\mathrm{E} u^{\prime}(x+c \varepsilon)}{u^{\prime \prime}(w-x)+\mathrm{E} u^{\prime \prime}(x+c \varepsilon) c}
$$

The denominator of this expression is negative by virtue of risk aversion and the numerator will be negative if $u^{\prime \prime \prime}>0$. Hence, under an assumption of decreasing absolute risk aversion, $x^{\prime}(c)>0$, a finding that is consistent with the equilibrium condition described above.

\section{An algebraic example}

Let us apply formula (11) to a simple problem involving a quadratic utility function and a discrete uniform distribution. We let $u(y)=b y-y^{2} / 2$, and let $\varepsilon$ have the distribution $\varepsilon=n$ with probability $1 / 2$ and $\varepsilon=-n$ with probability 1/2. The individual maximization problem has first-order conditions:

$$
-[b-(w-x)]+(c / 2)[b-(x+c n)+b-(x-c n)]=0,
$$

which can be solved for

$$
x=\frac{w+b(c-1)}{1+c} .
$$

Thus,

$$
x^{\prime}(c)=\frac{2 b-w}{(1+c)^{2}}
$$


We also have

$$
\mathrm{E} u^{\prime}(x+c \varepsilon) \varepsilon=\frac{1}{2}[b-(x+c n)] n-\frac{1}{2}[b-(x-c n)] n=c n^{2} .
$$

Using (11) we obtain a formula characterizing the optimal $c$ :

$$
(1-c) \frac{(2 b-w)^{2}}{(1+c)^{3}}=c n^{2}
$$

Note that there will be a unique $c$ that satisfies this equation. We can easily examine the effects of parameter changes on the choice of the optimal $c$ :

(i) as the size of the random income, $n$, rises, $c$ must fall, so the optimal tax rate rises;

(ii) as wealth increases, the left-hand side decreases, so $c$ must decrease the optimal tax rate increases; and

(iii) as risk aversion increases, $b$ must decrease, so the optimal tax must increase.

Table 1

\begin{tabular}{lllll}
\hline & & & $\begin{array}{l}\text { Utility } \\
\text { market }\end{array}$ & $\begin{array}{l}\text { Utility } \\
\text { insurance }\end{array}$ \\
\hline 0.1 & 0.93 & 0.48 & 0.745 & 0.745 \\
0.2 & 0.81 & 0.45 & 0.730 & 0.734 \\
0.3 & 0.69 & 0.41 & 0.705 & 0.720 \\
0.4 & 0.60 & 0.37 & 0.670 & 0.706 \\
0.5 & 0.52 & 0.34 & 0.625 & 0.691 \\
\hline
\end{tabular}

Table 1 presents some computations of the optimal tax for various values of the noise parameter $n$. In these computations we set $w=b=1$. The second and third columns of the table give the optimal values of $c$ and $x$. The optimal value of $D$ is of course $(1-c) x$; this implies that the demogrant as a percentage of income is simply $(1-c)$. The last two columns give the utility of the market (no intervention) case, and the social insurance case. By way of comparison, the utility of the full insurance case is 0.750 .

The main conclusion to be drawn from this table is that in this example the social insurance can capture only a small percentage of the gains possible from full insurance, unless the randomness in income is very extreme. In the case where $n=0.1$, for example, second-period income varies between 0.58 and 0.38 , but second-period consumption is smoothed out to vary between 0.57 and 0.39 - not a very big change, and consequently not a very big contribution to utility. 
One could argue that this inability of the linear tax to capture much of the potential gains is due to the overly restrictive assumption of linearity. A more flexible tax structure may capture more of the gains. Accordingly, in the next section, we investigate the problem of determining the optimal nonlinear tax.

\section{The optimal nonlinear tax}

In order to treat the nonlinear tax problem, it is convenient to rewrite (5)(7) introducing the density function for $\varepsilon$, which we denote by $f(\varepsilon)$. We suppose that $f(\varepsilon)$ is strictly positive over the interior of the compact interval $[a, b]$ and that $f(a)=f(b)=0$. Furthermore we change the variable of integration from $\varepsilon$ to $y=x+\varepsilon$, so that the limits of integration will now range from $y=x+a$ to $y=x+b$.

For reasons that will be explained later, we have to be careful about the domain of the utility function. We will suppose that utility is defined on the non-negative real line and that $x$ and $c(y)$ must lie in the compact region $0 \leqq x \leqq w$. With these changes eqs. (5)-(7) become:

$$
\max _{c(\cdot), x} u(w-x)+\int u(c(y)) f(y-x) \mathrm{d} y
$$

subject to

$$
\begin{aligned}
& x-\int c(y) f(y-x) \mathrm{d} y=0, \\
& -u^{\prime}(x-x)+\int u^{\prime}(c(y)) c^{\prime}(y) f(y-x) \mathrm{d} y=0, \\
& 0 \leqq x \leqq w ; \quad 0 \leqq c(y) \leqq w .
\end{aligned}
$$

It turns out to be convenient to integrate eq. (21) by parts to get

$$
u^{\prime}(w-x)+\int u(c(y)) f^{\prime}(y-x) \mathrm{d} y=0
$$

Here we have used the fact that $f(a)=f(b)=0$ and multiplied through by -1 . The resulting optimization problem, (19), (20), and (22), is a straightforward isoperimetric calculus of variations problem. It is, in fact, a problem of the same form as that considered by Mirrlees (1974).

In that work, Mirrlees used the Euler conditions of the calculus of variations to characterize the optimal solution to a similar problem. However, in the same paper, Mirrlees also showed that in some cases no optimal solution would exist. It is worthwhile reviewing his nonexistence argument here, as it applies to the social insurance problem. 
Let us choose consumption schedules of the form:

$$
\begin{aligned}
& c(y)=c_{1}, \quad \text { if } \quad y<\alpha \\
& =w / 2, \quad \text { if } \quad y \geqq \alpha n
\end{aligned}
$$

Here consumers receive the first-best level of consumption if their observed income exceeds some target level $\alpha$; if their observed income is less than $x$ they receive some (low) level of consumption $c_{1}$.

In order for this policy to be feasible it is sufficient to ensure that consumers will, on the average, choose to save $x=w / 2$. Mirrlees shows that if utility is unbounded below we can always choose $c_{1}$ low enough so as to induce this optimal level of savings.

Now consider what happens as we decrease the target level $\alpha$, but adjust $c_{1}$ so as to keep $x=w / 2$ and thus maintain feasibility. As $\alpha$ declines consumers have a higher and higher probability of ending up with the firstbest level of consumption; but if they are unlucky enough to end up with observed income less than $\alpha$, they will be more and more severely punished. The net impact of these two effects on expected utility is in general ambiguous but Mirrlees is able to show that in some cases total expected utility will increase as the target level $\alpha$ decreases. Hence he can construct a sequence of tax plans converging to the first-best optimum, but of course, never actually reaching it.

In this way, we can approximate as closely as we wish to the first-best optimum, by imposing penalties (presumably of great severity) on a small proportion of the population.

Although these farmers suffer severely, there are so few of them that their sufferings are outweighed by the encouragement their fate, or rather the prospect of it, gives to farmers taking production decisions. It seems that models of this kind can in certain cases provide some justification for extreme punishment of negligibly small groups [Mirrlees (1974)].

Thcre are two important problems raised by the Mirrlees result. The first is the question of the existence of an optimal tax. It seems clear that the problem here lies in Mirrlees's assumption of unbounded utility. If one imposes a bound on utility or consumption, on grounds of feasibility or humanitarian concern about ex post utility, the Mirrlees construction can only be carried so far - the unlucky consumers can only be punished to a certain degree. It seems possible that the imposition of such a bound would ensure the existence of an optimal tax.

However, the Mirrlees example raises another question. Even if the optimal tax exists when consumption is bounded, how do we know that it does not take the discontinuous form considered by Mirrlees? If it did take such a form. the Euler conditions used by Mirrlees would be irrelevant. 
In order to investigate these questions it is convenient to pose the optimization problem described by (19), (20), and (22) as a problem in optimal control. To do this we create two dummy state variables defined as:

$$
\begin{aligned}
& T(y)=\int_{x+a}^{y}[x-c(t)] f(t-x) \mathrm{d} t, \\
& R(y)=\int_{x+a}^{y}\left[u^{\prime}(w-x)+u(c(t))\right] f^{\prime}(t-x) \mathrm{d} t .
\end{aligned}
$$

Then we can write the relevant maximization problem as:

$$
\max _{x, c(y)} u(w-x)+\int u(c(y)) f(y-x) \mathrm{d} y
$$

subject to

$$
\begin{aligned}
& T^{\prime}(y)=[x-c(y)] f(y-x), \\
& R^{\prime}(y) \quad=\left[u^{\prime}(w-x)+u(c(y))\right] f^{\prime}(y-x), \\
& T(x+a)=T(x+b)=0, \\
& R(x+a)=R(x+b)=0, \\
& \subseteq \leqq c(y) \leqq \bar{c} ; \quad 0 \leqq x \leqq w,
\end{aligned}
$$

This is a straightforward problem in optimal control with the special features that (1) the Hamiltonian is independent of the state variables, and (2) the maximization takes place over a parameter $x$, as well as over the function $c(y)$. There are very general existence results for control problems with feature (1), and in the appendix I extend one of these results to the parameterized case. Hence, under quite weak assumptions an optimal tax schedule will exist.

A standard application of the maximum principle shows that at each income level $y, c(y)$ will maximize the Hamiltonian function over the allowable values of $c$. We pose this maximization problem here using $\lambda$ and $\mu$ to denote the adjoint variables:

$$
\begin{aligned}
\max _{c(y)} u(w-x)+u(c(y)) f(y-x)+\lambda[x-c(y)] f(y-x) & \\
& +\mu\left[u^{\prime}(w-x)+u(c(y)] f^{\prime}(y-x)\right]
\end{aligned}
$$

subject to

$$
\begin{aligned}
-c(y) & \leqq c, \\
c(y) & \leqq \bar{c} .
\end{aligned}
$$


Letting $\rho_{1}$ and $\rho_{2}$ be the Kuhn-Tucker multipliers on the inequality constraints we have the following conditions which characterize the optimal choice of $c(y)$ at each $y$ :

$$
\begin{aligned}
& u^{\prime}(c(y))\left[f(y-x)+\mu f^{\prime}(y-x)\right]=\lambda f(y-x)-\rho_{1}+\rho_{2}, \\
& c(y)=\underline{c}, \quad \rho_{1} \geqq 0, \\
& c<c(y)<\bar{c}, \quad \rho_{1}=\rho_{2}=0, \\
& c(y)=\bar{c}, \quad \rho_{2} \geqq 0 .
\end{aligned}
$$

We also have the first-order condition that $x$ maximizes the Hamiltonian. Using the original formulation in (19)-(21), this condition can be written as

$$
\lambda\left[1-\int c^{\prime}(y) f(y-x) \mathrm{d} y\right]-\mu \Delta(x)=0 .
$$

Here we have used the fact that the derivative of the objective function must vanish, owing to the individual optimization constraint, and we have let $\Delta(x)$ be the second derivative of individual utility as given in eq. (4).

We first establish the following technical result.

Proposition. If $\lambda \neq 0$ then $c(y)$ is continuous on the support of $f(\varepsilon)$.

Proof. According to Fleming and Rishel (1975, theorem 6.1), we need only establish that the Hamiltonian has a unique maximizer at each value of $y$.

Referring to the Kuhn-Tucker condition (33), we see that if $[f(y-x)+$ $\left.\mu f^{\prime}(y-x)\right] \neq 0$, the concavity of $u(c)$ guarantees a unique solution to (33). If the left-hand side of (33) is zero, then there are two cases: and

(i) $\lambda>0$; then since $f(y-x)>0$ and $\rho_{2} \geqq 0$, we must have $\rho_{1}>0$ and $c=\underline{c}$;

(ii) $\lambda<0$; by analogous reasoning, we must have $\rho_{2}>0$, and $c=\bar{c}$. Q.E.D.

This proposition shows that as long as the aggregate income constraint is binding, the optimal tax will be continuous on the support of $\varepsilon$. Thus, for at least some values of income, the optimal level of consumption will be in the interior of $[\underline{c}, \bar{c}]$ and the Euler conditions derived by Mirrlees will be relevant. In the interior case, we have a very simple formula characterizing $c(y)$ :

$$
u^{\prime}(c(y))=\frac{\lambda}{1+\mu h(y-\bar{y})} .
$$

where $h(\varepsilon)=f^{\prime}(\varepsilon) / f(\varepsilon)$ and $\bar{y}=\mathrm{E} y=\mathrm{E}(x+\varepsilon)=x$. Since $u^{\prime \prime}<0$, we can directly invert (38) to solve for $c(y)$, up to the constants $\lambda$ and $\mu$. 
We can use the information contained in eqs. (33)-(37) to provide some qualitative information concerning $\lambda$ and $\mu$. First, we note that at the modal value of $\varepsilon-$ call it $\hat{\varepsilon}=\hat{y}-\bar{y}-$ we have $f^{\prime}(\hat{\varepsilon})=0$, so

$$
u^{\prime}\left(c(\hat{y}-\bar{y})=\hat{\lambda}+\frac{\rho_{2}-\rho_{1}}{f(\hat{y}-\bar{y})} .\right.
$$

The complementary slackness conditions (34)-(36) show that if the mode occurs at a value of $\hat{y}$ where $c \leqq c(\hat{y})<\bar{c}$, then $\lambda$ will be positive. If $c<c(\hat{y})<\bar{c}$, then $\lambda$ is just the marginal utility of income at the modal value of $\varepsilon$.

In order to provide an expression for $\mu$, we rearrange (37) to get

$$
\mu=\frac{\left(1-\int c^{\prime}(y) f(y-\bar{y}) \mathrm{d} y\right) \lambda}{\Delta(x)} .
$$

The term in parentheses is simply the average marginal tax rate which we would generally expect to be positive. Since $\lambda>0$ by $(38)$ and $\Delta(x)<0$, since it is the second-order condition for individual maximization, we would generally expect $\mu$ to be negative.

In fact, it can be shown that $\mu<0$ under the assumption that $h^{\prime}(\varepsilon)<0$. To see this differentiate (38) with respect to $y$ to get:

$$
c^{\prime}(y)=\frac{-\lambda \mu h^{\prime}(y-\bar{y})}{u^{\prime \prime}(c(y))(1+\mu h(y-\bar{y}))^{2}} .
$$

Suppose, per absurdum, that $\mu>0$. Then (41) implies that $c^{\prime}(y)<0$. This in turn, by eq. (40) implies $\mu<0$. This contradiction then establishes three important facts: (1) $\mu<0,(2) c^{\prime}(y)>0$, and (3) the average marginal tax rate is positive. (Analogous facts were established by Mirrlees (1974) for the problem he considered; however, the additive structure of this problem allows for a considerable simplification of the arguments. ${ }^{4}$ )

The most interesting behavior of the optimal tax formula given by (38) is in the behavior of the marginal tax rate as a function of income. In the optimal taxation literature concerned with the equity-efficiency tradeoff arising because of unobserved differences of ability, it can be shown that the marginal tax rate on the highest observed income must be zero. This effect basically occurs because any tax schedule with a nonzero marginal tax rate at the high end can be Pareto dominated by one with a lower marginal tax rate - the highest income person is made better off by lowering his marginal tax rate and revenues available for redistribution are unaffected. [See Brito and Oakland (1977), Cooter (1978), Phelps (1973), Sadka (1976), and Seade (1977), among others.]

\footnotetext{
${ }^{4}$ Holmström (1979) also establishes analogous facts for a similar problem under somewhat different conditions.
} 
In the social insurance framework this effect is absent. Indeed, one would suspect the marginal tax rate on the highest income person might be quite large: if the only way to become a millionaire is to be lucky, there should be very small incentive losses from taxing a million dollar income at a high rate - and there are substantial insurance gains since one can then subsidize million dollar occurrences of bad luck.

Let us examine this argument more rigorously. First, if the $\bar{c}$ constraint is binding at high levels of income, the marginal tax rate is clearly one, so we concentrate on the interior case. Differentiating (38) we have:

$$
u^{\prime \prime}(c(y)) c^{\prime}(y)=\frac{-\lambda}{(1+\mu h(y))^{2}} \mu h^{\prime}(y) .
$$

Using (38) and rearranging:

$$
c^{\prime}(y)=\left[-\frac{u^{\prime}(c(y))}{u^{\prime \prime}(c(y))}\right] \frac{\mu}{\lambda} u^{\prime}(c(y)) h^{\prime}(y) .
$$

Letting $\rho(c(y))=-u^{\prime \prime}(c(y)) / u^{\prime}(c(y))$ be the Arrow-Pratt measure of absolute risk aversion, we have

$$
c^{\prime}(y)=\frac{1}{\rho(c(y))} \frac{\mu}{\lambda} u^{\prime}\left(c\left(y^{\prime}\right) h^{\prime}\left(y^{\prime}\right) .\right.
$$

This equation shows that, 'other things being equal', a higher degree of risk aversion implies a higher marginal tax rate. (Of course other things can never be equal since they also depend on the degree of risk aversion.)

Differentiating (44) once more we have

$$
c^{\prime \prime}(y)=\frac{\mu}{i \rho}\left[\left(h^{\prime} u^{\prime \prime} c^{\prime}+u^{\prime} h^{\prime \prime}\right)-\frac{\rho^{\prime}}{\rho} c^{\prime} u^{\prime} h^{\prime}\right] .
$$

The term in front of the brackets will generally be negative. If we are willing to assume $h^{\prime} \leqq 0, h^{\prime \prime} \geqq 0$ the first term inside the brackets will be positive which suggests a declining marginal consumption rate - i.e. an increasing marginal tax rate. The second term, however, works in the opposite direction. If absolute risk aversion decreases with income, the second term will be positive and the overall sign of $c^{\prime \prime}$ will be ambiguous. Clearly $\rho^{\prime} \leqq 0$ will imply $c^{\prime \prime}<0$, but this seems an implausible assumption on a priori grounds. However, it does seem clear that if risk aversion does not decline too rapidly, then $c^{\prime \prime}(y)$ will be negative and our earlier intuition will be supported. 
A nice example to illustrate these considerations is that of a constant relative risk aversion utility function and a normal distribution on $\varepsilon .{ }^{5}$ Since $u(c)=c^{1-\rho} /(1-\rho),(33)$ implies that

$$
c(y)=\left[\frac{1}{\lambda}+\frac{\mu}{\lambda} h(y)\right]^{1 / \rho}
$$

or

$$
c(y)=[B+A h(y)]^{1 / \rho},
$$

where $B>0, A<0$, and $h^{\prime}(y)<0$. In this case $\rho^{\prime}=0$ and the first term in (44) always dominates so that $c^{\prime \prime}(y)<0$.

If $\varepsilon$ is normally distributed with

$$
f(\varepsilon)=k \mathrm{e}^{-\varepsilon^{2} / 2},
$$

we have

$$
h(\varepsilon)=\frac{f^{\prime}(\varepsilon)}{f(\varepsilon)}=-\varepsilon,
$$

so that the optimal tax takes the form:

$$
c(y)=\left\{\begin{array}{l}
\underline{c}, \text { if } \subseteq \geqq[B-A y]^{1 / \rho} \\
{[B-A y]^{1 / \rho}} \\
\bar{c}, \text { if } \bar{c} \leqq[B-A y]^{1 / \rho} .
\end{array}\right.
$$

If $\rho=1$, a linear tax is optimal. As risk aversion increases, a more progressive tax is desirable, exactly as our intuition suggested.

The case with $\rho=1$ is an especially simple case in which to calculate optimal taxes. Table 2 presents an example of one such computation with $w=40, \sigma=2$ and 3 . As before, the columns labelled 'market', 'insurance', and 'full' give the utility from each arrangement.

Note the relatively low marginal tax rate. In the case where $\sigma=3$, income would lie in the two standard deviation range 14-26, but the marginal tax rate would be only 4 percent. The demogrant would also be 4 percent of first-period income. The partial insurance offers a 'small' improvement on the market solution, but does not capture much of the potential gains from complete insurance.

\footnotetext{
${ }^{5}$ Of course, for an optimal tax to exist we should truncate the distribution on $\varepsilon$ so that it has compact support.
} 
Table 2

\begin{tabular}{llllll}
\hline$\sigma$ & $c$ & $x$ & Market & Insurance & Full \\
\hline 2 & 0.98 & 19.897 & 5.9863 & 5.9864 & 5.9914 \\
3 & 0.96 & 19.815 & 5.9799 & 5.9804 & 5.9914 \\
\hline
\end{tabular}

\section{A curious example}

In some circumstances the optimal tax may take a rather peculiar form. Suppose, for example, that there are occurrences of $\varepsilon$ which reveal unambiguously the ex ante actions of the consumers. For example, suppose $\varepsilon$ has support $(a, b)$ and $w / 2+a>0$. Then if one observes mcasured income $y$ in the interval $w / 2+a>y>0$, one can be certain that the consumer saved less than $w / 2$ in the first period. In this circumstance it makes sense to choose a consumption schedule that would give the consumer a very low level of consumption in this region; so low, in fact, that the consumer would always save $x=w / 2$ so as to avoid falling into this region. In this way, we can support the first-best optimal solution. ${ }^{6}$

The mathematical details go as follows. As in section 5, let us choose a policy of the form:

$$
\begin{aligned}
& c(x+\varepsilon)=c_{1}, \quad \text { if } \quad x+\varepsilon<w / 2+a, \\
& w / 2, \quad \text { if } \quad x+\varepsilon \geqq w / 2+a .
\end{aligned}
$$

We want to choose $c_{1}$ small enough so that the utility from choosing $x=w / 2$ exceeds the utility from choosing any other choice of $x$. That is, we choose $c_{1}$ so that

$$
\begin{aligned}
2 u(w / 2)>\max _{x} u(w-x)+u(w / 2) & \\
+ & {\left[u\left(c_{1}\right)-u(w / 2)\right] F(w / 2+a-x), }
\end{aligned}
$$

where $F(\cdot)$ is the cumulative distribution function of $\varepsilon$.

For fixed $c_{1}$, the right-hand side of this inequality is bounded above, so a maximum exists. As long as $u\left(c_{1}\right)$ can be made sufficiently small, we can be assured of satisfying inequality (51) and thereby being able to support the first-best optimal pattern of consumption.

\footnotetext{
${ }^{6}$ Harris and Raviv $(1978 \mathrm{a}$, b) have independently noted that a first-best solution in principal agent problems often exists under similar circumstances.
} 


\section{Summary and conclusions}

If income contains a random component then a system of redistributive taxation will contribute to reducing the variance of after-tax income. The design of an optimal program of redistributive taxation must weigh the benefits from this 'social insurance' against the deadweight costs of a tax on transactions. In this paper we have examined the determination of such an optimal tax in a simple choice theoretic framework.

In the case of linear taxation the optimal tax can be derived from a relatively simple and intuitive formula. The nonlinear tax involves a less intuitive but surprisingly explicit formula. We have shown that if one bounds the allowable values of consumption the optimal tax will always exist and generally be continuous as a function of observed income. Under reasonable assumptions about the distribution of the randomness in income, the optimal tax will be increasing in income and the average marginal tax rate will be positive. The optimal tax may exhibit an increasing marginal tax rate, depending on the nature of the stochastic influences on income.

Finally it should be remarked that the analysis of this paper can be applied to a number of other problems involving moral hazard and insurance. In particular, the existence and continuity results can be easily generalized to the principal--agent problems considered by Harris and Raviv (1978a, b), Ilolmström (1979), and Shavell (1975).

\section{Appendix}

\section{Existence of an optimal solution}

Here we state an existence theorem for a class of optimal control problems that is referred to in the text. Consider the control problem:

$$
\max _{u(\cdot), z} \int_{t_{0}}^{t_{1}} h_{0}(t, u(t), z) \mathrm{d} t
$$

subject to

$$
\begin{aligned}
& \begin{array}{l}
\mathrm{d} x \\
\mathrm{~d} t
\end{array}=h(t, u(t), z), \\
& x\left(t_{0}\right)=x_{0}(z) ; \quad x\left(t_{1}\right)=x_{1}(z), \\
& u(t) \text { in } \Omega, \quad \text { a compact subset of } R^{n}, \\
& z \text { in } Z, \quad \text { a compact subset of } R^{n}, \\
& x(t) \text { in } R^{n}, \\
& x_{0}, x_{1}, h_{0} \text { and } h \text { continuously differentiable. }
\end{aligned}
$$


Here $x$ is the vector of state variables, $u$ is the vector of control variables, and $z$ is a vector of parameters. We assume that a feasible solution exists. For fixed $z$, this is a standard control problem with the special feature that $h_{0}$ and $h$ are independent of the state variables. Theorem 6.3 of Berkovitz (1974, p. 165) shows that for fixed $z$ an optimal solution to this control problem exists. We wish to extend this argument to the parameterized case. First we introduce some notation.

Given an admissable control $u(\cdot)$ and a parameter $z$, consider the system of differential equations given by

$$
\begin{aligned}
& \frac{\mathrm{d} y}{\mathrm{~d} t}=h_{0}(t, u(t), z), \\
& \frac{\mathrm{d} x}{\mathrm{~d} t}=h(t, u(t), z), \\
& y\left(t_{0}\right)=0, \\
& x\left(t_{0}\right)=x_{0}(z) .
\end{aligned}
$$

The 'attainable set' (for fixed $z$ ) is defined to be the set of states of this system at $t_{1}$ as $u(\cdot)$ ranges over the set of admissible controls.

$$
\begin{aligned}
K_{z}= & \left\{(y, x) \in \mathbf{R}^{n+1}: y=y\left(t_{1}\right) ; \quad x=x\left(t_{1}\right),\right. \\
& \text { where } y(\cdot) \text { and } x(\cdot) \text { solve }(\text { A.3) for some admissible control } \\
& u(\cdot),\}
\end{aligned}
$$

Note that $y=y\left(t_{1}\right)$ is simply the value of the objective function in problem (A.1). Berkovitz (1974, theorem 6.3) shows that $K_{z}$ is compact, so clearly $y$ achieves a maximum value on $K_{z}$. Hence an optimal solution to the control problem with fixed $z$ and no boundary constraint at $t_{1}$ exists.

For the parameterized problem the analogous attainable set is:

$$
K=\left\{(y, x, z):(y, x) \in K_{z}, z \in Z\right\}
$$

This is clearly compact since it is the product of two compact sets.

Finally, we incorporate the additional boundary constraint by defining:

$$
K^{\prime}=K \cap\left\{(y, x, z) \in K: x=x_{1}(z)\right\}
$$

This set is compact since it is a closed subset of $K$; it is nonempty by hypothesis. Hence $y$ attains a maximum on $K^{\prime}$ and a solution to (A.1) and (A.2) therefore exists. 


\section{References}

Atkinson, A. and J. Stiglitz, 1976, The design of tax structure: Direct versus indirect taxation, Journal of Public Economics 6, 55-76.

Berkovitz, L., 1974, Optimal control theory (Springer-Verlag, New York).

Beveridge, W., 1942, Social insurance and allied services (Macmillan, New York).

Brito, D. and W. Oakland, 1977, Some properties of the optimal income tax, International Economic Review 407-424.

Coe, Richard, 1977, Dependence and poverty in the short and long run, Survey Research Center, University of Michigan.

Diamond, P., 1975, A many person Ramsey tax rule, Journal of Public Economics 4, 335-342.

Diamond, P. and J. Mirrlees, 1971, Optimal taxation and public production I-II, American Economic Review 61, 8--27, 261-278.

Diamond, P. and J. Mirrlees, 1978, A model of social insurance with variable retirement, M.I.T. Working Paper no. 210.

Diamond, P., J. Helms and J. Mirrlees, 1978, Optimal taxation in a stochastic economy, A Cobb-Douglas cxamplc, M.1.T. Working Paper no. 217.

Fleming, W. and R. Rishel, 1975, Deterministic and stochastic optimal control (Springer-Verlag, New York).

Harris, M. and A. Raviv, 1978a, Some results on incentive contracts, American Economic Review 68, 20-30.

Harris, M. and A. Raviv, 1978b, Optimal incentive contracts with imperfect information, Journal of Economic Theory 20, 231-259.

Holmström, B., 1979, Moral hazard and observability, Bell Journal of Ecc. omics 10, 74-91.

Jencks, C., 1972, Inequality (Basic Books, New York).

Lillard, L. and R. Willis, 1978, Dynamic aspects of earnings mobility. Econometrica 46, 985 1012.

Mirrlees, J., 1971, An exploration in the theory of optimum income taxation, Review of Economic Studies 38, 175 208.

Mirrlees, J., 1974, Notes on welfare economics, information, and uncertainty, in: Balch, McFadden and $\mathrm{Wu}$, Essays on economic behavior under uncertainty (North-Holland, Amsterdam).

Mirrlees, J., 1975, On moral hazard and the theory of unobservable behavior, Oxford University, mimeo.

Phelps, E., 1973, Taxation of wage income for economic justice, Quarterly Journal of Economics $87,331-354$.

Ross, S., 1979, quoted in Family Weekly, June 24.

Sadka, E., 1976, On income distribution, incentive effects, and optimal income taxation, Review of F.conomic Studies 43, 261-267.

Sandmo, A., 1976, Optimal taxation - An introduction to the literature, Journal of public economicś 6, 37-54.

ieade, J., 1977, On the shape of optimal tax schedules, Journal of Public Economics 7, 203-236.

$\therefore$ avell, S., 1975, Risk sharing and incentives in the principal and agent relationship, Bell Iournal of Economics 10, 5573 . 